\title{
Method of gas detection applied to an infrared hyperspectral sensor
}

\author{
Mariusz Kastek, ${ }^{* 1}$ Tadeusz Piatkowski, ${ }^{1}$ Rafał Dulski, ${ }^{1}$ Martin Chamberland, ${ }^{2}$ \\ Philippe Lagueux ${ }^{2}$ and Vincent Farley ${ }^{2}$ \\ ${ }^{1}$ Institute of Optoelectronics, Military University of Technology, Kaliskiego 25, 00-908 Warsaw, Poland \\ ${ }^{2}$ Telops Inc., Quebec, Canada
}

Received October 14, 2012; accepted December 19, 2012; published December 31, 2012

\begin{abstract}
This paper presents the detection and identification of gases using an infrared imaging Fourier-transform spectrometer. The principle of operation of the spectrometer and the method for gases detection and identification has been shown in the paper. The variation of a signal reaching the IFTS caused by the presence of a gas has been calculated and compared with the reference signal obtained without the presence of a gas in IFTS's field of view. Some results of the detection of various types of gases have been presented too.
\end{abstract}

The problem of remote detection of chemical substances appears in many, sometimes extremely different fields of human activities. The applications of detection devices include the monitoring of technological processes, diagnostics of industrial installations, monitoring of natural environment and military purposes. Such diversity has caused the development of many detection methods employing various physical phenomena due to which detection and identification of chemical compounds are possible. Among these methods, a significant group constitutes the solutions using the phenomenon of selective absorption of electromagnetic radiation by a chemical compound. Here, we can distinguish the solutions based on absorption of laser radiation emitted by an illuminator, being an element of a measuring system, and thermovision methods recording thermal radiation. The methods of the first group are active methods employing the absorption of laser radiation by chemical compounds. Thermovision methods use the absorption bands of chemical compounds in the IR spectrum.

The present paper deals with the remote gas detection, identification and quantification from turbulent stack plumes with an IFTS method. It presents first the modeling that is required in order to get an appropriate understanding of both the scene and the instrument. Next it covers the methodology of the developed detection and identification approach. Finally, results are presented to demonstrate the capabilities and the performance of the remote gas detection and identification by using hyperspectral data obtained from the Telops Hyper-Cam

*E-mail: makstek@wat.edu.pll spectrometer. The latter is described in much detail in the references [1-4].

The infrared imaging Fourier-transform spectrometer (IFTS) - Hyper-Cam LWIR - used on experiment was built by Telops Inc.. This IFTS use 320x256 pixel Mercury Cadmium Telluride (MCT) focal plane arrays (FPA) with a $6^{\circ} \times 5^{\circ}$ FOV. The FPA has a Stirling cooler to provide good noise figures in a field ready package. Spectral information is obtained using a technique called Fourier Transform Infrared Radiometry (FTIR). FTIR is a classical interference based technique applied to gas spectroscopy that uses a Michelson interferometer to mix an incoming signal with itself at several different discrete time delays. The resulting time domain waveform, called an interferogram, is related to the power spectrum of the scene through the Fourier transform. An interferogram for each pixel in an image is created by imaging the output of the interferometer onto a focal plane array and collecting data at each discrete time delay. Advantages of using an FTIR sensor over a filter or grating based system include higher resolution for equal cost and the absence of misalignment of different color images due to platform motion. However, FTIR does have the disadvantage of producing a slower frame rate than filter based systems because twice as many points are taken for the same number of spectral points. For atmospheric tracking of gases, however, the LWIR sensor has a sufficient frame rate [4].
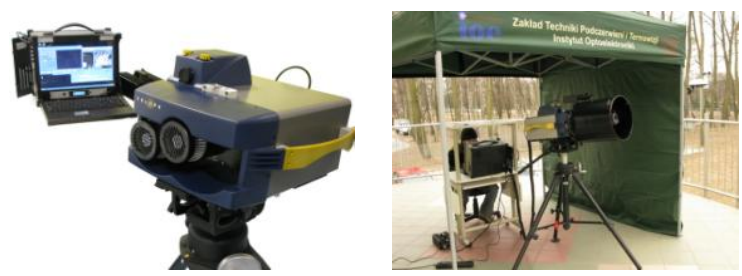

Fig. 1. The infrared imaging Fourier-transform spectrometer (IFTS) Hyper-Cam.

Data for this collection can be taken from $0.25 \mathrm{~cm}^{-1}$ to $150 \mathrm{~cm}^{-1}$ spectral resolution between $830 \mathrm{~cm}^{-1}(12 \mu \mathrm{m})$ and $1290 \mathrm{~cm}^{-1}(7.75 \mu \mathrm{m})$ at a frame rate of $0.2 \mathrm{~Hz}$. In addition to the infrared data, visible imagery was taken using a 
firewire camera that is boresighted to the IR sensor. Figure 1 shows the picture of a Hyper-Cam LWIR. The sensor is controlled by a field computer and data is stored on a RAID drive to guarantee integrity of data [4].

The main difference between the standard and imaging interferometer is the application of focal plane array (FPA) detector type. The imaging device can be described as a classical interferometer multiplied by the number of pixels in the array. As a result, the spectral analysis of interferograms at single pixels gives the spectral information of the entire image. The spectral distribution for an entire image is obtained by performing the analysis according to Eq. (2) for every pixel of an FPA array. The results can be treated as 3-dimensional data (two image coordinates and a wavelength) and analyzed using special software for detection and identification of gases.

The target detection and identification algorithm is generally based on three key factors: the composition of the analyzed pixel, the type of model used to estimate the variability of the target and background spaces, and the model used to describe the pure and mixed pixels. The mathematical representation of a mixed pixel depends on whether the background (or target) space is estimated statistically or geometrically. The considered sub-pixel target detection algorithms are of a stochastic nature. When the background is entirely represented by its statistics, the detection problem consists in extracting the targeted spectral signatures from a background noise term: $\varepsilon_{b}$. Equations (1) and (2) express this symbolically:

$$
\begin{gathered}
H_{0}: x=\varepsilon_{b} \\
H_{1}: x=T_{a}+\varepsilon_{b}
\end{gathered}
$$

Here, $a$ is a weighting vector to be estimated and $T$ is a matrix of " $k$ " targets: $\left[t_{1}, t_{2}, t_{3}, \ldots, t_{k}\right]$. It is assumed that the noise component has a mean value of zero and covariance $\boldsymbol{C}$.

The basic idea behind the clutter-matched filter (CMF) is to minimize the response to the unknown background signatures while accentuating the response to the target spectrum. To do so, the following mathematical operator was developed:

$$
q_{i}=\frac{C^{-1} t_{i}}{\sqrt{t^{\prime}{ }_{i} C^{-1} t_{i}}}
$$

where $q_{i}$ is the CMF related to the $i^{\text {th }}$ target $t_{i}$ taken from the matrix of targets $T$ and the $\mathrm{N} \times \mathrm{N}$ estimated covariance matrix, $C$, is given by following equation:

$$
C=\frac{1}{(M-1)} \sum_{i=1}^{M}\left(x_{i}-\mu\right)\left(x_{i}-\mu\right)^{\prime}
$$

Here, $x_{i}$ and $\mu$ are respectively the spectrum of the $i^{\text {th }}$ pixel and the mean pixel of the scene and $M$ is the number of pixels from the image used to estimate the covariance. This model falls under the stochastic methods category.

The signal to clutter ratio is simply calculated with the use of the following equation:

$$
\frac{\text { Signal }}{\text { Clutter }}=q \cdot\left(x_{i}-\mu\right)
$$

If the CMF is not calculated for each scene, which is sensible if real-time processing is required, then the mean pixel found in Eq. (4) must be the one introduced in Eq. (5) when the covariance matrix was introduced.

The CMF is normalized so that, when the signal is absent, the probability density function of the filtered image has a standard deviation of 1 . The score of each pixel denotes the number of standard deviations that this pixel parts from zero. As can be ascertained with Eq. (3), the response of the CMF is linearly dependant on the target signature. The results obtained with this model give an approximate concentration of the targeted species occupying the pixels. Effectively, the scores obtained when the CMF is applied are a good indicator of the strength of the signal, which, in turn, is proportional to the temperature contrast and to the column density of the target.

When the target mean is known, it is possible to use the spectral angle mapper (SAM, or, as it will sometimes be called in this article, the correlation coefficient) to detect the presence of targeted species. As its name indicates, SAM is an algorithm that computes the angle formed by the projection of a target on an image pixel:

$$
x_{i} \cdot t=\left\|x_{i}\right\|\left\|t_{i}\right\| \cos (\theta)
$$

or, in a more common form:

$$
\cos (\theta)=\frac{x_{i} \cdot t}{\left\|x_{i}\right\|\|t\|}
$$

The results are limited between 0 and 1 . A value of 0 signifies that the image pixel and the target are not at all correlated whereas a score of 1 means that the two vectors are perfectly correlated. SAM is obviously not a linear filter and it cannot indicate the strength of the signal. The detection and identification of chemical agent is performed through post-processing of the recorded hyperspectral. The algorithm integrates a double-stage decisional scheme used to analyze the hyperspectral data produced by Hyper-Cam sensor. Measurement of the background is performed to obtain the covariance matrix of the scene. This covariance is used to calculate the clutter-matched filters for several gas signatures from a library based on PNNL data. On each scene measurement, the background is removed and the calculation of the image of clutter-matched filter scores and correlation (SAM) scores is performed. To detect and identify a given 
chemical agent, the score from both calculations (CMF and SAM) must exceed a given threshold [5].

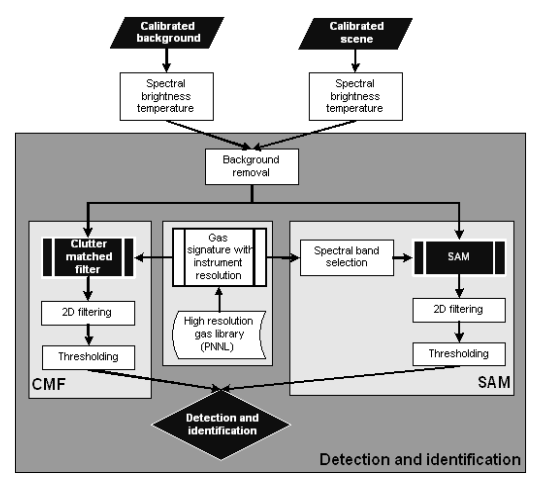

Fig. 2. Double-stage detection and identification of scheme used to process hyperspectral data.

During the field tests the following gases were measured: $\mathrm{CO}_{2}$, propane-butane mixture and freon $134\left(\mathrm{CH}_{2} \mathrm{FCF}_{3}\right.$ tetrafluoroethane). In each case several gas concentrations were tested in order to verify the efficiency of gas measurements in the open space by a Hyper-Cam LWIR sensor.

Freon 134 was the gas used during the field measurements. This gas exhibits absorption bands in the long wave infrared range, thus it can be detected using Hyper-Cam LWIR. Registrations were made at two distances: $60 \mathrm{~m}$ and $20 \mathrm{~m}$, for two gas concentrations of $3 \%$ and $6 \%$, respectively. During the field measurements the weather conditions (humidity, pressure, wind speed and direction) were monitored by an automatic weather station Vantage Pro. It was observed that wind speed has the most significant influence on the measurement results. On the basis of the experience gained during the laboratory measurement sessions and previous measurements and theoretical analyses [6], it can be estimated that the thermal contrast $\Delta \mathrm{T}$ (between gas cloud and background) above $3 \mathrm{~K}$ significantly increases the probability of gas detection by a Hyper-Cam device. The results are presented in Fig. 3. The Matlab procedure applied for the analysis of data from Hyper-Cam utilized the reference signature database and CMF module (Clutter Matched Filter) [7].

The background scene is shown on the broadband IR images with an overlay of the detected and identified chemicals. The overlay has a variable transparency. Where the scores from both the CMF and the SAM are above the thresholds, the transparency is displayed and it is proportional to the score of the CMF. This gives an indication of the concentration of the chemical and this also clearly displays the cloud shape. The reference signatures of gases were adopted from PNNL database. The algorithm used for the gas detection, identification and quantification is still under development and the presented results are preliminary. However, as can be seen from Fig. 4, the data processing by CMF module successfully reveals the presence of gas (marked by a red color) [7].
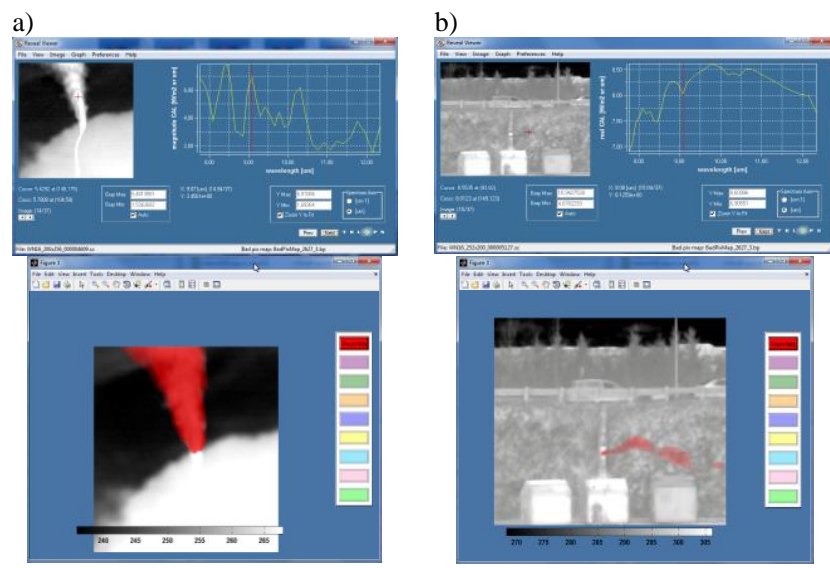

Fig. 3. The results of freon 134 measurements (concentration 3\%, Tatm $=18^{\circ} \mathrm{C}$, vwind $=8 \mathrm{~m} / \mathrm{s}$, patm $=992 \mathrm{hPa}$ ): (a) distance $20 \mathrm{~m}$, (b) distance $60 \mathrm{~m}$.

IFTS technique is a very useful method for gas detection in the open space. Its sensitivity is of an order of magnitude lower than typical laboratory methods, mainly due to the fact that lock-in detection is not possible. However, the possibility to present the results in the form of an image is a clear advantage, because the gas can be not only detected, but also located in space. Even its lower accuracy is still sufficient to detect and identify particular gases in a mixed gas cloud. The measurements conducted by means of IFTS spectrometers have lower accuracy due to lower sensitivity, the influence of weather conditions (mainly wind speed) and unknown thickness of a visualized gas cloud. Currently, the new quantification algorithm is under development, in which the cloud thickness will be automatically estimated on the basis of spatial distribution of areas with emissivity different than that of a background. This new method combining mathematical analysis, data from reference signature database (PNNL database), CMF (Clutter Matched Filter) module and additionally SAM (Spectral Angle Map) module should improve the effectiveness of gas detection by IFTS-based solutions.

\section{References}

[1] V. Farley, M. Chamberland et al., Proc. SPIE 6661, 66610L (2007).

[2] A. Vallières, A. Villemaire et al., Proc. SPIE 5995, 59950G (2005).

[3] M. Chamberland, C. Belzile et al., Proc. SPIE 5416, 63 (2005).

[4] V. Farley, C. Belzile et al., Proc. SPIE 5546, 29 (2005).

[5] P. Tremblay, S. Savary et al., Proc. SPIE 7673, 76730H (2010).

[6] M. Kastek, T. Sosnowski et al., WIT Transactions on Ecology and the Environment 123, 227 (2009).

[7] M. Kastek, T. Piątkowski, P. Trzaskawka et al., Metrol. Meas. Syst. XVIII, 4 (2011). 